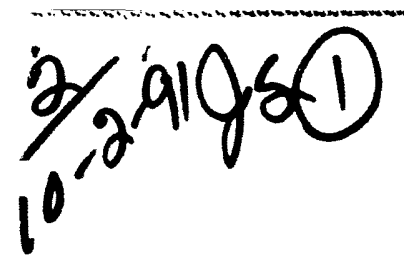

UCRL-I D--106094

DE92 000093

\title{
An Analysis of Grazing Incidence lietal Mirrors \\ in a Laser ICF Reactor Driver
}

Robert Bieri

Michael Guinan

July 12, 1991

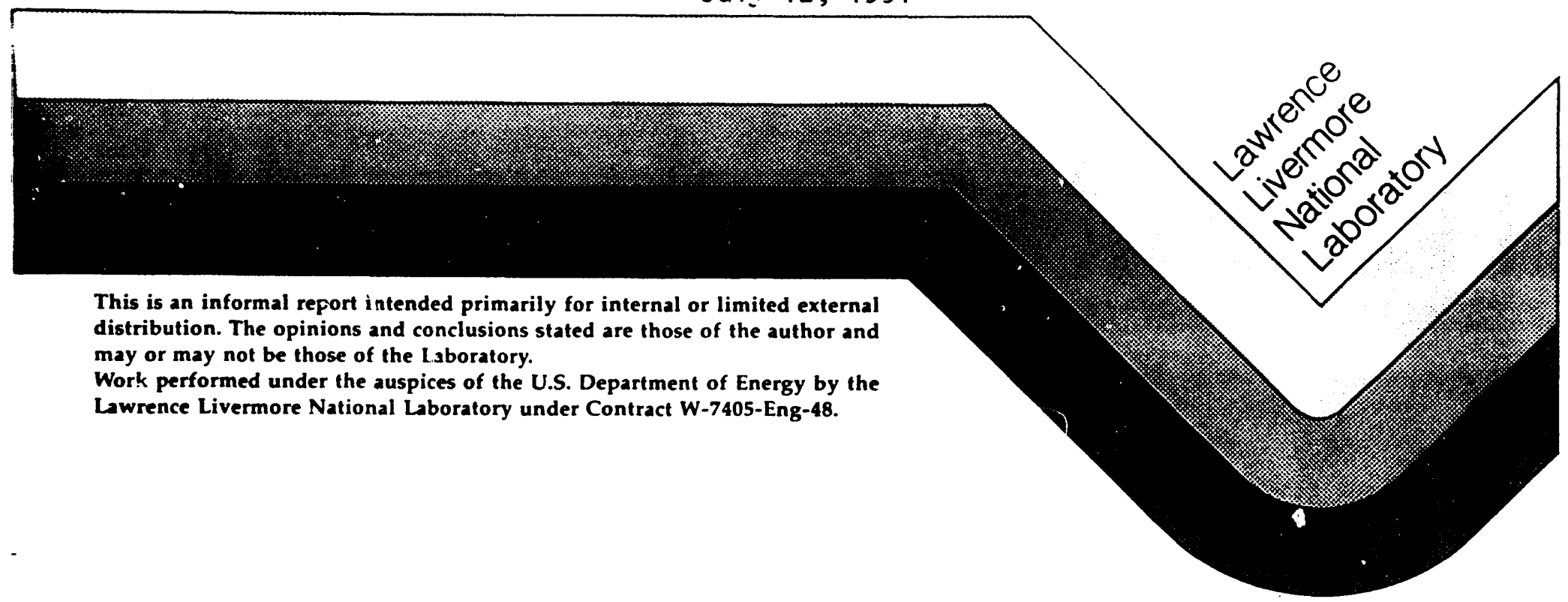




\section{DISCLAIMER}

This document was prepared as an acccount of work sponsored by an agency of the United States Government. Neither the United States Government nor the University of California nor any of their employees, makes any warranty, express or implied, or assumes any legal liability or responsibility for the accuracy, completeness, or usefulness of any information, apparatus, product, or process disclosed, or represents that its use would not infringe privately own rights. Reference herein to any specific commercial products, process, or service by trade name, trademark, manufacturer, or otherwise, does not necessarily constitute or imply its endorsement, recommendation, or favoring by the United States Government or the University of California. The views and opinions of authors expressed herein do not necessarily state or reflect those of the United States Government or the University of California, and shall not be used for advertising or product endorsement purposes.

This report has been reproduced directly from the best available copy.

Available to DOE and DOE contractors from the Office of Scientific and Technical Information P.O. Box 62, Oak Ridge, TN 37831

Prices available from (615) 576-8401, FTS 626-8401

Available to the public from the National Technical Information Service

U.S. Department of Commerce 5285 Port Royal Rd. Springfield, VA 22161 


\title{
An Analysis of Grazing Incidence Metal Mirrors in a Laser ICF Reactor Driver
}

\author{
Robert Bieri \\ Michael Guinan
}

June 18, 1991 


\begin{abstract}
Grazing incidence metal mirrors (GIMMs) are examined to replace dielectric mirrors for the final elements in a laser beam line for an inertial confinement fusion reactor. For a laser driver with a wavelength from 250 to $500 \mathrm{~nm}$ in a 10-ns pulse, irradiated mirrors made of $\mathrm{Al}, \mathrm{Al}$ alloys, or $\mathrm{Mg}$ were found to have calculated laser damage limits of $0.3-2.3 \mathrm{~J} / \mathrm{cm}^{2}$ of beam energy and neutron lifetime fluence limits of over $5 \times 10^{20} 14 \mathrm{MeV} \mathrm{n} / \mathrm{cm}^{2}$ (or 2.4 full power years when used in a $1,000-\mathrm{MW}$ reactor) when used at grazing incidence (an angle of incidence of 85 degrees) and operated at room temperature or at $77 \mathrm{~K}$. A final focusing system including mirrors made of $\mathrm{Al}$ alloy 7475 at room temperature or at liquid nitrogen temperatures used with a driver which delivers $5 \mathrm{MJ}$ of beam energy in 32 beams would require 32 mirrors of roughly $10 \mathrm{~m}^{2}$ each. This chapter includes calculations of damage limits for GIMMs and discusses critical issues relevant to the integrity and lifetime of such mirrors in a reactor environment.

The reflectivities of various metals are calculated from measured optical constants at room temperature and at cryogenic temperatures ( $\geq 77 \mathrm{~K}$ ) for 250 - to $500-\mathrm{nm}$ light at both normal (highest reflectivities $>90 \%$ ) and grazing incidence (highest reflectivities $>99 \%$ ). Then,for the mirrors in a representative system ( 32 beams, $5 \mathrm{MJ}$ total energy, 10-ns pulse), the thermal absorption and conduction rates of the best candidate metals (magnesium, aluminum, and aluminum alloys) are used with the maximum allowable cyclic thermal stress to give the maximum allowed surface-temperature rise and surface thermal load. The allowed surface thermal load and surface reflectivity give the maximum beam energy density (optical damage threshold) and the minimum size for each mirror. For mirrors made of aluminum alloy 7475 and initially operated at room temperature, the resulting optical damage threshold and allowable temperature rise give a required mirror size for each final mirror. Critical issues relevant to the integrity and lifetime of such mirrors in a reactor environment are briefly discussed.
\end{abstract}




\section{Introduction}

A 5-MJ laser producing a 1000-MJ thermonuclear yield will result in approximately $3.6 \times 10^{20} 14 \mathrm{MeV}$ neutrons per shot; at a distance of $50 \mathrm{~m}$ from the target (the location of the final dielectric mirror) this corresponds to a fluence of $1.1 \times 10^{12} \mathrm{n} / \mathrm{cm}^{2}$ for each shot. Since the last focusing or turning element of each beam cannot be shielded from these neutrons, it must be made of a material which can withstand the lifetime fluence associated with these radiation levels. If multilayer mirrors cannot withstand neutron irradiation, a grazing incidence metal mirror (GIMM), similar to those studied for use in free-electron lasers [1], could be placed between the last dielectric mirror and the target. Figures 1 and 2 diagram an inertial confinement fusion (ICF) laser driver whose use of metal mirrors[2] allows the more sensitive optical elements to be located out of direct line-of-sight of the neutrons. In this geometry, the metal mirror is located $30 \mathrm{~m}$ from the target, and the dielectric mirror is $20 \mathrm{~m}$ behind the GIMM and 10 degrees removed from the direction of line-of-sight neutrons. Neutron transport calculations indicate that this geometry provides the more sensitive optical components with a sufficiently reduced neutron exposure to ensure an affordable component lifetime.

Grazing incidence metal mirrors are shown to have sufficiently high reflectivities to be attractive candidates for the final elements of a laser driver for an ICF reactor. 


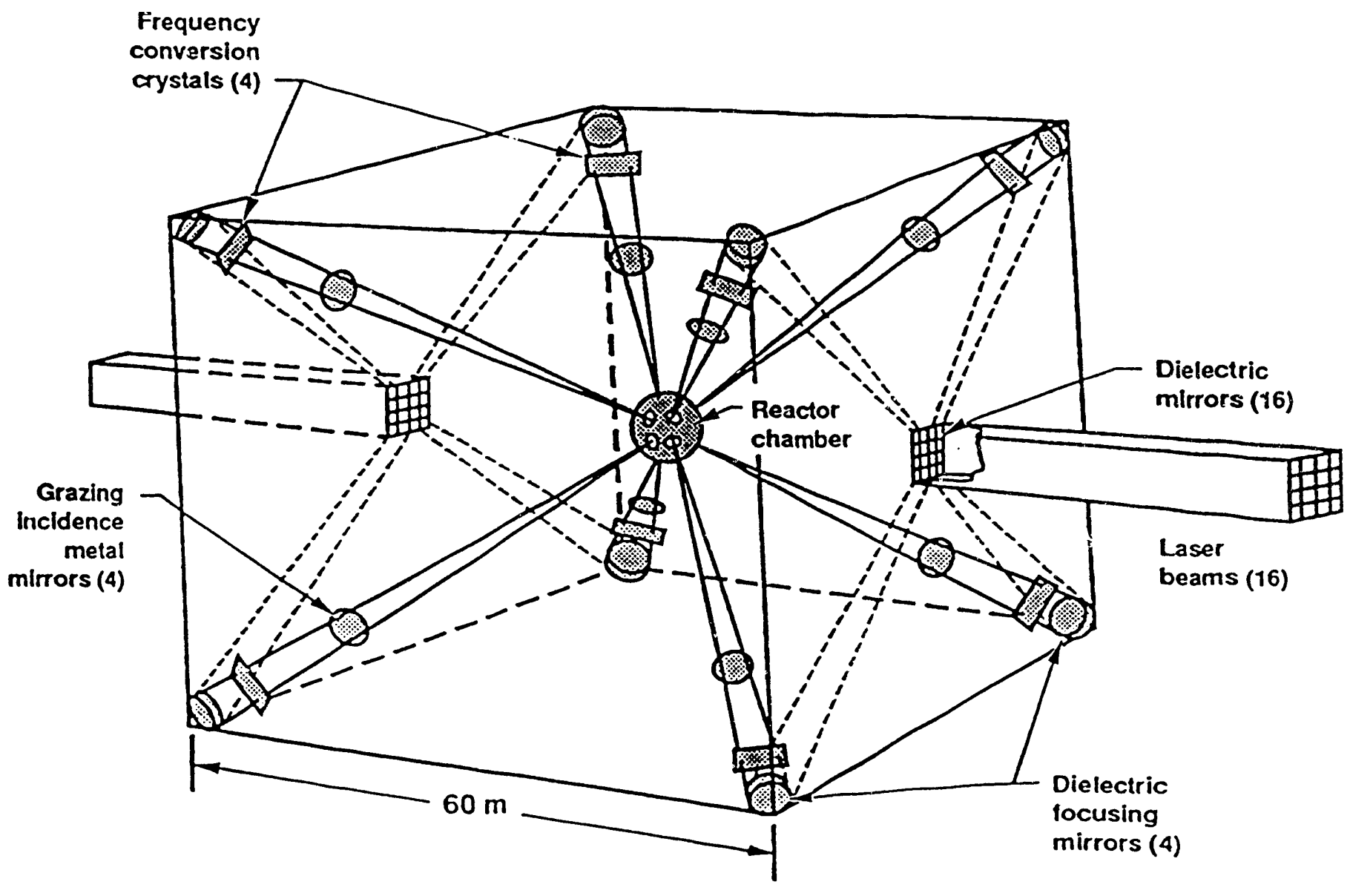

Figure 1: Final focusing spaceframe for a laser driven ICF reactor 


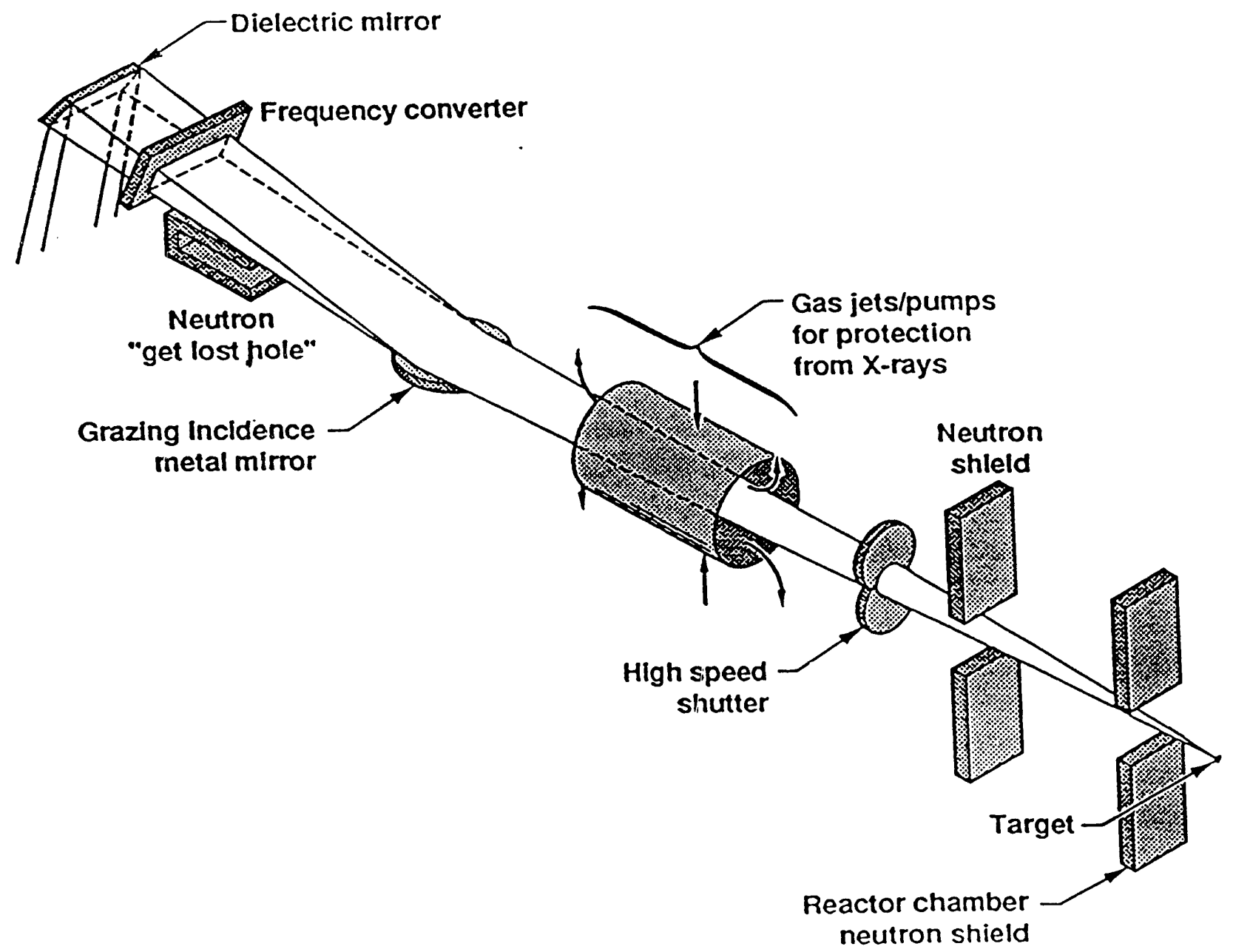

Figure 2: Final beam transport elements

\section{Protection of Sensitive Optics}

Neutron transport calculations have been done to address several issues concerning how effectively the GIMMs shield the remaining optical elements of the driver[3]. The calculations include the neutron flux, spectrum, and energy deposition for the GIMM, the dielectric turning mirror, a KDP nonlinear optic crystal (if required for frequency conversion), liquid nitrogen to cool the mirror (if low temperature operation is required), and 1 Torr- 
meter of argon used to attenuate soft x-rays. The implications for the activation of beam-line components were also examined.

\subsection{Effect of Neutrons on Multilayer Dielectric Mir- rors}

Neutron radiation can destroy dielectric multilayer mirrors by degrading the optical transmission of the dielectric layers, by chemical decomposition of the dielectrics, or by destroying the boundaries between layers. Transmission degradation data is sparse, but measurements[4] for $\mathrm{MgF}_{2}$ and $\mathrm{ZnS}$ show an order-of-magnitude degradation in absorption or transmission after $10^{16} \mathrm{n} / \mathrm{cm}^{2}$ (or about 1 hour of operation for an unshielded dielectric $50 \mathrm{~m}$ from a 1,000-MWe reactor) for wavelengths of interest (250-500 $\mathrm{nm}$ ). This damage may be removable through continuous annealing, and dielectrics may exist which have color centers far removed from wavelengths of interest. Unfortunately, even if there is no loss of transmissive properties, the multilayer mirror can still be compromised by neutron damage. All ionic dielectrics will undergo significant radiolysis[5], radiation-induced chemical decomposition, after energy depositions of about $1 \mathrm{eV} /$ atom, or $5 \times 10^{17} \mathrm{n} / \mathrm{cm}^{2}$ for $\mathrm{MgF}_{2}$; a few $\mathrm{eV} /$ atom energy deposition is also enough to cause significant amorphization in $\mathrm{SiO}_{2}$. For dielectric materials which are more resistant to damage, chemical mixing at the interfaces will still be a problem. Any collisional cascade at an interface will cause mixing of the two dielectrics and create a thin, possibly amorphous, third phase region with unknown optical properties. Collisional mixing at the boundaries will occur over a thickness of roughly $3 \mathrm{~nm} /(\mathrm{DPA})^{1 / 2}\left(1 \mathrm{DPA} \simeq 5 \times 10^{20} 14 \mathrm{MeV} \mathrm{n} / \mathrm{cm}^{2}\right.$ for most dielectrics, which corresponds to about 1 full power year for a 1,000-MWe plant), and enhanced diffusion will cause mixing over a thickness of roughly $30 \mathrm{~nm} /(\mathrm{DPA})^{1 / 2}[6]$. If the very existence of a third phase does not destroy the optical properties of a multilayer mirror, a change of thickness of only $\lambda / 16$ in a $\lambda / 4$ layer will destroy the constructive interference required for high reflectivities. Under the most optimistic assumptions, the best conceivable unshielded multilayer mirror would probably only last a fraction of a full power year. 


\subsection{Results of Neutron Transport Calculations}

Neutron flux

The results of the TART 3-D neutron transport code indicate that the neutron flux at the final optics is almost entirely from neutrons that shine directly on the GIMM. There will be significant contributions to the flux at the final turning mirror (and frequency converter if present) from scattered $14-\mathrm{MeV}$ neutrons and from lower energy backscattered neutrons; less than $10 \%$ of the neutrons arriving at the sensitive optics will have energies $\geq 1$ $\mathrm{MeV}$. There is little or no data on neutron damage to dielectric mirrors, but if we make the conservative assumption that a multilayer mirror with no color centers will have a lifetime fluence limit of $10^{17}-10^{18}$ neutrons $/ \mathrm{cm}^{2}$ for neutrons with energies above $1 \mathrm{MeV}$, we can estimate the lifetime of a dielectric mirror. The total neutron flux at the turning mirror and the frequency converter, if present, is $1-2 \times 10^{10}$ neutrons $/\left(\mathrm{cm}^{2} \mathrm{~s}\right)$. Since only $10 \%$ of these have energies above $1 \mathrm{MeV}$, the lifetime of the sensitive optics will be from 2-20 years. If there were no GIMM, the flux at a turning mirror $50 \mathrm{~m}$ from the target would be $10^{12} \mathrm{n} / \mathrm{cm}^{2}$ and consist almost entirely of high energy neutrons; this flux would give a lifetime of only 1 day under our damage assumptions.

\section{Activation}

Because of the activation of $\mathrm{Al}$ and $\mathrm{Mg}$, the mirrors will have to be within a shielded area. $\mathrm{A} \mathrm{Mg}$ mirror would require 17 days of cool-down before it could be worked on; an $\mathrm{Al}$ mirror would require remote or limited maintenance because of the long lived $\mathrm{Al}^{26}$ that is formed after 10 days of operation. Waste disposal and accidental release are not a problem if the metal mirrors are replaced every 1-2 years. 


\section{Reflectivities and Absorptances of Graz- ing Incidence Metal Mirrors}

The reflectivity of a conducting metal is a function of the wavelength and polarization of the incident light and the angle at which the light strikes the surface of the metal. Since laser drivers include polarization, we can orient the mirrors so that the incident light has the polarization (transverse electric) which produces the highest reflectivity. The reflection coefficient for a transverse electric (TE) polarized wave is given by [7]:

$$
r_{12}=\left(n_{1} \cos \theta_{1}-n_{2} \cos \theta_{2}\right) /\left(n_{1} \cos \theta_{1}+n_{2} \cos \theta_{2}\right)
$$

where $\theta_{i}$ is the angle of incidence of the light in medium $i$.

For a conductor, $n_{2} \cos \theta_{2}=u+i v$, and the reflectivity is given by

$$
R=\left|r_{12}\right|^{2}=\frac{u^{2}+v^{2}-2 u \cos \theta_{i}+\cos ^{2} \theta_{i}}{u^{2}+v^{2}+2 u \cos \theta_{i}+\cos ^{2} \theta_{i}}
$$

for

$$
\begin{aligned}
& 2 u^{2}=\left(n^{2}-k^{2}-\sin ^{2} \theta_{1}\right)+\left[\left(n^{2}-k^{2}-\sin ^{2} \theta_{1}\right)^{2}+4 n^{2} k^{2}\right]^{1 / 2} \\
& 2 v^{2}=-\left(n^{2}-k^{2}-\sin ^{2} \theta_{1}\right)+\left[\left(n^{2}-k^{2}-\sin ^{2} \theta_{1}\right)^{2}+4 n^{2} k^{2}\right]^{1 / 2}
\end{aligned}
$$

where $n$ and $k$ are the frequency dependent refractive index and extinction coefficient of the metal. 


\subsection{Reflectivities Calculated from Experimental Data}

The undamaged grazing incidence reflectivities for $\mathrm{Al}$ and $\mathrm{Mg}$ calculated from experimental values $[8,9]$ for $n$ and $k$ for $250 \mathrm{~nm}$ light are shown in Table 1. The leading candidate metals, aluminum $[10,11,12]$ and magnesium [12], give reflectivities of over $99 \%$ for an angle of incidence of $85^{\circ}$; the normal incidence $\left(\theta_{i}=0\right)$ room-temperature reflectivity for both metals for light from 2.5 to $5.0 \mathrm{eV}$ (250 to $500 \mathrm{~nm}$ ) ranges from 92 to $95 \%$. Although lower reflectivities can be compensated for by using larger mirrors, a grazing incidence mirror with less than $99 \%$ reflectivity would have to be too large to be of use in a reasonable illumination geometry. Table 2 lists normal and grazing incidence reflectivities for photon energies of interest (2.5 to $5.0 \mathrm{eV}$ ) for aluminum, silver, and magnesium.

Although silver also has nearly as high a reflectivity at the longer wavelengths $[13,14,15,16]$, it is less attractive for several reasons. Neutron irradiation can cause significant surface roughening of silver (due to dense collision cascades that do not occur in aluminum or magnesium) which will decrease its reflectivity. There are also absorption bands due to interband transitions [17] and surface plasmons $[18,19]$ that are very close to the desired operating wavelength. In addition, defected silver films show an additional absorption band [20] in the wavelength region of interest. 
Table 1: Normal and grazing incidence reflectivities for various metals

\begin{tabular}{||c|c|c|c|c|c||}
\hline $\mathrm{Metal}$ & Wavelength $(\mathrm{nm})$ & $\mathbf{n}$ & $\mathrm{k}$ & $\mathrm{R}_{\text {normal }}(\%)$ & $\mathrm{R}_{\text {Graz.Inc. }}(\%)$ \\
\hline $\mathrm{Al}$ & 250 & 0.183 & 2.88 & 92.45 & 99.36 \\
$\mathrm{Al}$ & 500 & 0.771 & 5.91 & 91.90 & 99.28 \\
$\mathrm{Ag}$ & 332 & 0.41 & 0.65 & 31.97 & 94.20 \\
$\mathrm{Ag}$ & 350 & 0.12 & 1.35 & 84.40 & 98.82 \\
$\mathrm{Ag}$ & 400 & 0.075 & 1.93 & 93.85 & 99.51 \\
$\mathrm{Au}$ & 257 & 0.92 & 1.14 & 26.19 & 90.36 \\
$\mathrm{Au}$ & 450 & 1.4 & 1.88 & 39.75 & 92.66 \\
$\mathrm{Be}$ & 405 & 2.48 & 2.2 & 41.48 & 92.71 \\
$\mathrm{Cd}$ & 280 & 0.41 & 2 & 72.61 & 97.52 \\
$\mathrm{Co}$ & 275 & 1.41 & 1.52 & 30.53 & 90.68 \\
$\mathrm{Cu}$ & 347 & 1.19 & 1.47 & 31.58 & 91.13 \\
$\mathrm{Cu}$ & 450 & 0.87 & 2.2 & 58.26 & 95.71 \\
$\mathrm{Fe}$ & 440 & 2.94 & 3.34 & 55.92 & 95.11 \\
$\mathrm{In}$ & 420 & 0.651 & 1.85 & 57.54 & 95.76 \\
$\mathrm{Ir}$ & 220 & 0.74 & 2.01 & 58.12 & 95.76 \\
$\mathrm{Mg}$ & 250 & 0.111 & 2 & 91.52 & 99.31 \\
$\mathrm{Mg}$ & 400 & 0.184 & 3.66 & 95.03 & 99.57 \\
$\mathrm{Mo}$ & 312 & 2.02 & 3.01 & 55.56 & 95.12 \\
$\mathrm{Mo}$ & 366 & 2.43 & 2.97 & 52.78 & 94.67 \\
$\mathrm{Ni}$ & 275 & 1.09 & 1.26 & 26.79 & 90.14 \\
$\mathrm{Ni}$ & 440 & 1.567 & 2.68 & 54.57 & 95.03 \\
$\mathrm{~Pb}$ & 589 & 2.01 & 3.48 & 62.02 & 96.00 \\
$\mathrm{Pt}$ & 257 & 1.17 & 1.93 & 44.51 & 93.61 \\
$\mathrm{Pt}$ & 441 & 1.94 & 6.13 & 83.21 & 98.43 \\
$\mathrm{Rh}$ & 400 & 0.84 & 3.91 & 82.01 & 98.33 \\
$\mathrm{Rh}$ & 546 & 1.62 & 4.63 & 77.10 & 97.80 \\
$\mathrm{Se}$ & 320 & 2.57 & 1.16 & 27.01 & 89.17 \\
$\mathrm{Se}$ & 340 & 2.661 & 1.06 & 26.73 & 89.06 \\
$\mathrm{Si}$ & 500 & 4.3 & 0.74 & 39.94 & 92.25 \\
$\mathrm{Sn}$ & 587 & 1.48 & 7.77 & 91.10 & 99.20 \\
$\mathrm{Steel}$ & 325 & 1.37 & 2.09 & 45.12 & 93.62 \\
$\mathrm{Zn}$ & 326 & 0.599 & 2.23 & 68.16 & 96.96 \\
$\mathrm{Zn}$ & 361 & 0.72 & 2.61 & 70.52 & 97.17 \\
\hline \hline
\end{tabular}


Table 2: Dependence of reflectivities on photon energy

\begin{tabular}{|c|c|c|c|c|}
\hline \multicolumn{5}{|c|}{ Aluminum } \\
\hline Photon Energy (eV) & $\mathbf{n}$ & $\mathbf{k}$ & $\mathrm{R}_{\text {normal }} \%$ & $\mathrm{R}_{\text {Graz.Inc. } \%}$ \\
\hline 2.29 & 0.82 & $\overline{5.99}$ & 91.63 & 99.25 \\
\hline 2.5 & 0.771 & 5.91 & 91.90 & 99.28 \\
\hline 2.54 & 0.64 & 5.5 & 92.23 & 99.31 \\
\hline 3 & 0.548 & 5 & 92.00 & 99.29 \\
\hline 5.47 & 0.34 & 3.8 & 91.62 & 99.26 \\
\hline 3.5 & 0.353 & 3.93 & 91.83 & 99.28 \\
\hline 3.68 & 0.31 & 3.56 & 91.38 & 99.25 \\
\hline 4 & 0.298 & 3.7 & 92.25 & 99.32 \\
\hline 5 & 0.183 & 2.88 & 92.45 & 99.36 \\
\hline \multicolumn{5}{|c|}{ Silver } \\
\hline Photon Energy (eV) & $\mathbf{n}$ & $\mathbf{k}$ & $\mathrm{R}_{\text {normal }} \%$ & $\mathrm{R}_{\text {Graz.Inc. } \%}$ \\
\hline 2.5 & 0.237 & 3.09 & 91.44 & 99.26 \\
\hline 3 & 0.226 & 2.28 & 86.51 & 98.85 \\
\hline 3.25 & 0.229 & 1.86 & 81.57 & 98.44 \\
\hline 3.5 & 0.211 & 1.42 & 75.77 & 98.03 \\
\hline 3.6 & 0.23 & 1.13 & 67.02 & 97.39 \\
\hline 3.7 & 0.3 & 0.765 & 47.26 & 95.94 \\
\hline 3.77 & 0.527 & 0.399 & 15.37 & 92.48 \\
\hline 3.8 & 0.727 & 0.304 & 5.43 & 87.37 \\
\hline 3.9 & 1.295 & 0.36 & 4.01 & 74.75 \\
\hline 4 & 1.615 & 0.599 & 10.24 & 81.73 \\
\hline 4.1 & 1.729 & 0.845 & 15.26 & 84.93 \\
\hline 4.2 & 1.752 & 1.06 & 19.42 & 86.88 \\
\hline 4.3 & 1.729 & 1.13 & 20.73 & 87.43 \\
\hline 4.5 & 1.694 & 1.28 & 23.83 & 88.57 \\
\hline 4.75 & 1.612 & 1.34 & 25.18 & 89.06 \\
\hline 5 & 1.294 & 1.336 & 26.55 & 89.78 \\
\hline \multicolumn{5}{|c|}{ Magnesium } \\
\hline Photon Energy (eV) & $\mathbf{n}$ & $\mathbf{k}$ & $\mathrm{R}_{\text {normal }} \%$ & $\mathrm{R}_{\text {Graz.Inc. } \%}$ \\
\hline 2 & 0.382 & 5.74 & 95.62 & 99.62 \\
\hline 3 & 0.184 & 3.66 & 95.03 & 99.57 \\
\hline 4 & 0.132 & 2.63 & 93.56 & 99.46 \\
\hline 5 & 0.111 & 2 & 91.52 & 99.31 \\
\hline
\end{tabular}


Figure 3 shows the normal incidence reflectivity of silver, and Figure 4 shows the normal incidence reflectivities of aluminum and magnesium. As Figure 3 shows from 2.48 to $3.51 \mathrm{eV}$ (500 to $353 \mathrm{~nm}$ ), we are operating very close to the plastive plase for silver, and at $5 \mathrm{eV}(250 \mathrm{~nm})$, the reflectivity has dropped to $25 \%$. For magnesium and aluminum, we are well below the plasma edges and above the interband transition lines $(\simeq 1.5 \mathrm{eV}$ $\mathrm{fc}_{\overline{1}}$ aluminum and $\simeq 0.75$ for magnesium). Therefore, we will exclude silver from further consicieration as a viable candidate material. Some aluminum alloys also have high enough reflectivities to be of interest and are discussed in later sections of this paper.

Figure 5 shows the reflectivity at $85^{\circ}$ angle of incidence as a function of photon energy for both aluminum and magnesium. At photon energies of 2.5 to $5.0 \mathrm{eV}$, we are well removed from the various absorptive features.

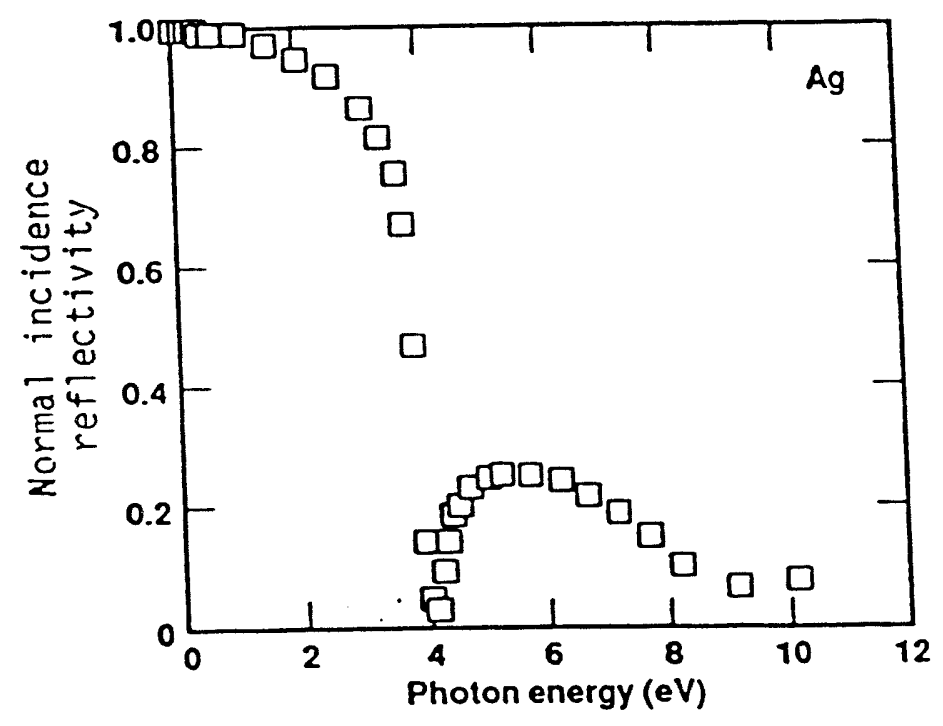

Figure 3 Normal incidence reflectivity of silver 


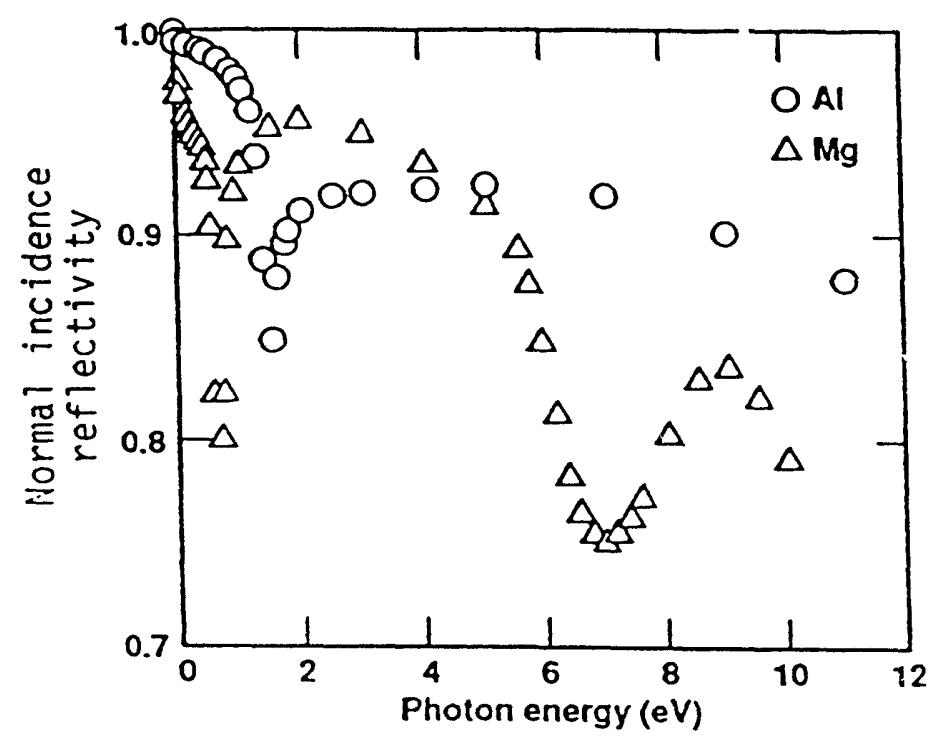

Figure 4 Normal incidence reflectivities of $\mathrm{Al}$ and $\mathrm{Mg}$

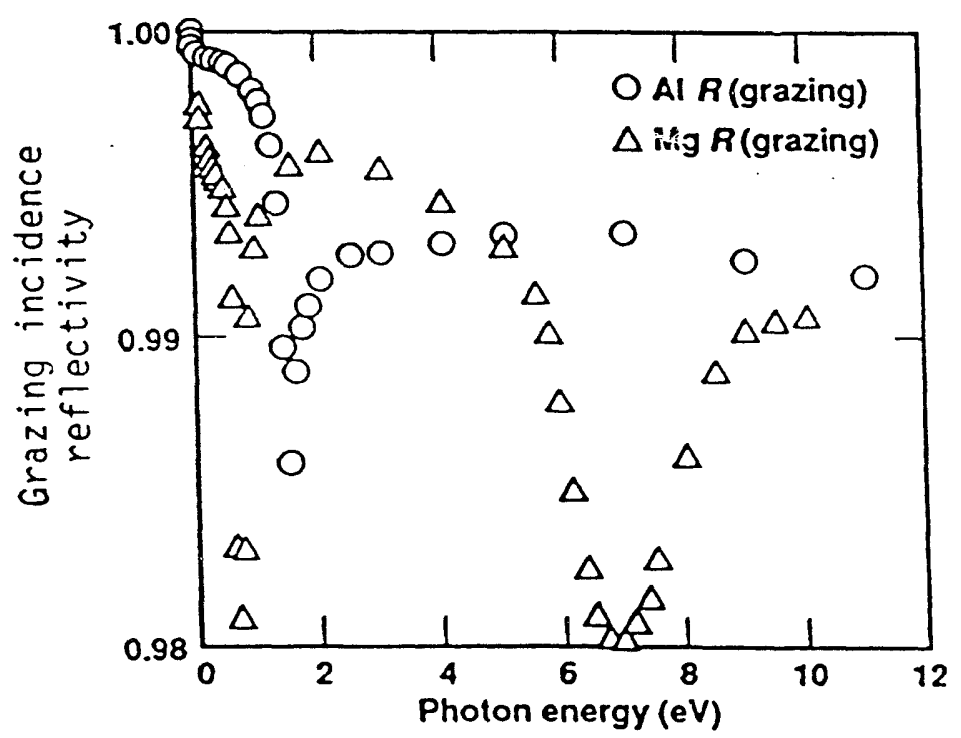

Figure 5 Grazing incidence reflectivities of $\mathrm{Al}$ and $\mathrm{Mg}$ 


\subsection{Contributions to the Absorptance of Metals}

For our purposes, it is useful to discuss the absorptance, $A=1-R$, rather than the reflectivity of the metals. For free-electron metals, the absorptance is given by the Drude model $[21,22]$, which involves only two parameters: $\Gamma$, the electron relaxation frequency, and $\omega_{p}$, the metal plasma frequency. For the laser frequencies, $\omega$, and metals of interest, the inequalities

$$
\Gamma \stackrel{\sim}{<} \stackrel{\sim}{<} \omega_{\mathbf{p}}
$$

are satisfied. For this case, the opticial absorptance at normal incidence is

$$
A \simeq \frac{2 \Gamma}{\omega_{\mathrm{p}}}=\frac{\rho \omega_{\mathrm{p}}}{2 \pi}
$$

where $\rho$ is the dc resistivity in electrostatic units. The last equality is the classical result for magnesium or aluminum with $\omega_{\mathrm{p}}=\left(4 \pi n e^{2} / \mathrm{m}\right)^{1 / 2}=$ $2.26 \times 10^{16} \mathrm{~s}^{-1}$ for aluminum [12], $1.60 \times 10^{16} \mathrm{~s}^{-1}$ for magnesium [23], and $\rho_{\mathrm{Al}}=3.1 \times 10^{-18} \mathrm{~s}, \rho_{\mathrm{Mg}}=4.8 \times 10^{-18} \mathrm{~s}$ at $20^{\circ} \mathrm{S}[24]$. The above equation gives $A=1.12 \%$ for aluminum and $1.22 \%$ for magnesium.

For root-mean-square (rms) roughnesses of 1 to $2 \mathrm{~nm}$, electron scattering at the surface becomes diffuse, and the absorption of the surface increases due to the anomalous skin effect. The anomalous skin effect [25] will give an additional surface contribution to the absorptance, $A_{\mathbf{B}}$, given by:

$$
A_{\mathrm{s}} \simeq(3 / 4) v_{\mathrm{f}} / c=0.35 \%(\mathrm{Al} \text { or } \mathrm{Mg}),
$$

where $c=$ the velocity of light, and $v_{\mathrm{f}}=$ the Fermi velocity. Although it is possible to finish surfaces with rms roughnesses of about $1 \mathrm{~nm}$, surface sputtering would create rms roughnesses of more than $1 \mathrm{~nm}$ on the smoothest surface by removing a few atomic layers of the mirror. The mirror damage limit must therefore include absorptance contributions from the anomalous skin effect. 
We note that the experimental absorptances at normal incidence implied in Table 2, $8 \%$ for aluminum and $5-8 \%$ for magnesium, are much larger than the sum of the classical and surface absorptions listed above. This difference arises from frequency-dependent contributions to $\Gamma$ at short wavelengths. As photon energies increase, it becomes possible to excite electrons in lowerlying bands, which gives rise to interband absorption peaks. For photon energies near the absorption edges, there can also be contributions to the absorptance from phonon - assisted transitions. The presence of interband transitions also contributes to changes in the real part of the dielectric constant, which increase absorptance. These contributions are negligible at 2.5 to $5.0 \mathrm{eV}$ for magnesium but make a small contribution at $2.5 \mathrm{eV}$ for aluminum, which has an absorptance peak at $1.5 \mathrm{eV}$. Frequency-dependent contributions to $\Gamma$ can also arise from electron-electron interactions and deviations from free-electron bands. For a discussion of these effects, see the referenced books by Sokolov and Wooten $[25,22]$.

We can write the total absorptance as a sum of four terms:

$$
A=A_{0}+A_{T}+A_{S}+A_{\omega}
$$

where the first two terms correspond to the dc value of $\Gamma, A_{0}$ arising from imperfections and $A_{T}$ from the intrinsic dc resistivity. $A_{\omega}$ is the net result of all frequency-dependent contributions to $\Gamma$, and $A_{S}$ was given earlier. $A_{0}$ and $A_{S}$ will be independent of temperature, while $A_{T}$ will be proportional to the absolute temperature above $T=T_{\theta} / 2$, where $T_{\theta}$ is the Debye temperature of the mirror material. Below $T_{\theta} / 2, A_{T}$ will approach a constant value, given by $2 / 5 A_{T}\left(T=T_{\theta}\right)$, even though the dc resistivity continues to decrease. This is the result of quantum effects on the electron - phonon interaction at optical frequencies [26]. At a fraction of a micron, the contributions to $A$ from the first three absorptance terms are expected to increase with temperature as $A_{T}$, while $A_{\omega}$ is expected to be approximately temperature independent. Estimates of laser damage thresholds can be very sensitive to the temperature dependence of the absorptance, since the mirror temperature increases rapidly during the pulse. 


\section{Radiation Damage to Metal Mirrors}

Successful operation of a magnesium or aluminum mirror located $30 \mathrm{~m}$ from a $1000-\mathrm{MJ}, 3-\mathrm{Hz}$ pellet is theoretically possible. The mirror must be shielded from $\alpha$ particles, other charged particles, short-ranged neutral particles, macroscopic pellet debris, and $x$-rays. For high $\rho \mathrm{R}$ targets, less than one torr-meter of argon provides sufficient shielding for everything except the slow-moving pellet debris, which must be stopped by a high-speed shutter. Neutron damage will increase the optical absorptance by a factor of at most two, thus lowering the laser-damage threshold by a factor of approximately two. Overall swelling, melting, vaporization, surface erosion, creep, and dimensional and mechanical instability, as well as increased absorptance from transmutation products and neutron-induced defects, are expected to be tolerable.

Neutron radiation can compromise the first mirror in three ways:

1) The laser damage thyeshold may be lowered by increased resistivity of the metal dus to defects, transmutations, and surface roughening on an atomic scale.

2) The laser damage threshold may be lowered by increased absorptance of the mirror due to microscopic surface roughening.

3) The focusing of the mirror can deteriorate due to macroscopic distortions from swelling or creep of the mirror and support structure.

The first effect raises the normal incidence absorptance by $1 \%$ and the grazing incidence absorptance by $0.5 \%$; in both cases a contribution to the absorptance of $0.35 \%$ is due to the anomalous skin effect resulting from roughening of the surface on an atomic scale.

Damage from the second effect is negligible. The total sputtering from neutrons[27] and scattered argon[28] will be only $30 \mathrm{~nm} / \mathrm{year}$, with the variance in the surface due to sputtering considerably smaller. 
The third effect will be lifetime limiting for a room-temperature mirror, but will not be a concern for a cryogenic mirror made of $\mathrm{Al}$ or $\mathrm{Mg}$. There will be negligible saturation s'welling or creep in an $\mathrm{Al}$ or $\mathrm{Mg}$ mirror at cryogenic temperatures since the vacancies are immobile and saturation occurs. If the $7 \mathrm{~m} \times 1.8 \mathrm{~m}$ final mirror is conservatively limited to nonuniform swelling differences of $\lambda / 4$, the allowed volumetric swelling is $1.5 \%$ and the resulting lifetime fluence limits for $14-\mathrm{MeV}$ neutrons for room-temperature mirrors made of pure $\mathrm{Al}$, pure $\mathrm{Mg}$, and $\mathrm{Al} 7475$ are $2.5 \times 10^{20}, 8 \times 10^{19}$, and $5 \times 10^{20} \mathrm{n} / \mathrm{cm}^{2}$ respectively. The corresponding mirror lifetimes in a 1,000 MWe plant are $1.2,0.4$, and 2.4 full power years.

\subsection{Optical Requirements for a Grazing Incidence Mir- rors}

Although the grazing incidence requirements on extremely localized distortions of the mirror surface are more restricting than at normal incidence, the limits on macroscopic deviations are less stringent. The rms wave-front error for $\lambda / 4$ peak-to-valley figure errors in a normal incidence mirror is $\simeq 0.175$, bu ${ }^{\prime}<0.03$ for $\theta_{i}>80$ degrees for a grazing incidence mirror [29].

\subsection{Increase in Optical Absorptance}

Absorption can be increased by damage centers within the optical absorption depth of a metal. Because vacancies and interstitials are mobile at room temperature in both aluminum and magnesium, only the larger clustered defects remain to affect the absorptance of the mirror. Experimentally, less than $1 \%$ of the damage-induced resistivity remains at room temperature, and changes in absorptance are negligible. At low temperatures, however, the increase in optical absorptance resulting from neutron damage within the optical absorption depth of the reflector surface (several times the skin depth) remains and lowers the optical damage limit of the mirror. 
At liquid helium temperatures where all defects are immobile, the resistivity damage rate will be given by:

$$
\frac{d \rho}{d \phi}=K \rho_{\mathrm{f}} \sigma_{\mathrm{d}}
$$

where $K$ is an efficiency factor that takes into account recombinations occurring within the collision cascade, $\rho_{\mathrm{f}}$ is the resistivity increment per atom fraction of Frenkel pairs, and $\sigma_{d}$ is the displacement cross section. For neutrons, $\sigma_{\mathrm{d}}=\left\langle\sigma E_{\mathrm{D}}\right\rangle /\left(2 T_{\mathrm{A}}\right)$ is the simplest model. This substitution gives:

$$
\frac{d \rho}{d \phi}=K \rho_{\mathrm{f}}<\sigma E_{\mathrm{D}}>/\left(2 T_{\mathrm{A}}\right)
$$

where $\left\langle\sigma E_{\mathrm{D}}\right\rangle$ is the spectrum-averaged damage energy cross section, and $T_{\mathrm{A}}$ is the average displacement threshold (typically tens of eV). Damage energy cross sections have been calculated for a large number of metals in various neutron spectra including high-energy spectra [30].

The value of $K$ must be determined experimentally from measurements of $d \rho / d \phi$. To date, measurements at $2 \mathrm{~K}$ with $14-\mathrm{MeV}$ neutrons have been made on eleven materials: $\mathrm{Zr}, \mathrm{V}, \mathrm{Fe}, \mathrm{Nb}, \mathrm{Mo}, \mathrm{W}, \mathrm{Al}, \mathrm{Cu}, \mathrm{Ni}, \mathrm{Au}$, and Pt. However, for all materials, the value of $K$ was found to be the same as in fission reactor irradiations [31]. Therefore, the value of $K$ for magnesium was taken from reactor irradiations, and $d \rho / d \phi$ was calculated for $14-\mathrm{MeV}$ neutrons. Table 3 lists the results. The values used for $\rho_{\mathrm{f}}$ and $T_{\mathrm{A}}$ are from Jung [32].

Table 3: Resistivity changes due to 14 - MeV neutrons.

\begin{tabular}{||l|c|c|c||}
\hline \hline Metal & $\begin{array}{c}\frac{d \rho}{d \phi} \\
\left(10^{-25} \Omega \mathrm{cm}^{3}\right)\end{array}$ & $\begin{array}{c}\rho_{\text {saturation }} \\
\left(10^{-4} \mathrm{~W} \times \mathrm{cm}\right)\end{array}$ & $\begin{array}{c}\left(\frac{d \rho}{d \phi}\right)_{T R} \\
\left(10^{-25} \Omega \mathrm{cm}^{3}\right)\end{array}$ \\
\hline Magnesium & 12.1 & 1.1 & $1.5 \times 10^{-4}$ \\
Aluminum & 4.1 & 1.0 & $1.8 \times 10^{-4}$ \\
\hline
\end{tabular}


As irradiation proceeds, $d \rho / d \phi$ will decrease due to overlap of damaged regions. Since interstitials recombine with vacancies within a region of about 100 atomic volumes, the resistivity will saturate. Depending on the chosen model, $90 \%$ of saturation will be reached at fluences from 3 to 4 times the saturation fluence value that the initial rate would have given. The saturation resistivity, $\rho_{\mathrm{S}}$, for each metal $[33,34]$ is also listed in Table 3.

Because of the high defect mobility in aluminum and magnesium at room temperature, no resistivity change in either metal is expected if irradiation is performed at room temperature. Since interstitials in both metals are more mobile at $77 \mathrm{~K}$ than at $4 \mathrm{~K}$, defect saturation will occur at higher neutron fluences for irradiation at $77 \mathrm{~K}$ than for irradiation at $4 \mathrm{~K}$.

Even allowing for errors in $\rho_{\mathrm{S}}$ and for the additional $0.35 \%$ absorption from $A_{S}$, the change in $A$ at normal incidence at $77 \mathrm{~K}$ will be less than $1 \%$ and will be less than $0.5 \%$ at grazing incidence. Saturation will occur in $\sim 1$ month at $30 \mathrm{~m}$. To avoid frequent mirror replacements, we must increase the size of the mirro: to allow operation with the increased absorptance.

\section{Transmutation Products:}

Impurities will also be produced by $14-\mathrm{MeV}$ neutrons ( $\mathrm{Mg}$ in $\mathrm{Al}$ for example). From the known resistivity changes due to these products and the production cross sections, expected resistivity increments have been calculated and are included in Table 3 as $(d \rho / d \phi)_{\mathrm{TR}}$. The values indicate that transmutation products will not begin to contribute until fluences reach $10^{22} \mathrm{n} / \mathrm{cm}^{2}$. Measured changes of absorptance in aluminum alloyed with magnesium and lithium show large changes in the interband absorptance, but from 2.5 to $5.0 \mathrm{eV}$, changes in absorptance can be calculated from the resistivity changes [35]. 


\subsection{Neutron Damage to the Mirror Surface}

\section{Sputtering:}

Neutron sputtering is expected to be negligible for mirrors from a macroscopic point of view. However, there will be sufficient roughening to cause the electrons in the metal to scatter diffusely from the surface. Theoretical $14 \mathrm{MeV}$ neutron forward sputtering rates are 2 to $3 \times 10^{-5}$ sputtered atoms per incident neutron, assuming no cascade overlap [36]. With cascade overlap, the theoretical rate could be a factor of 3 greater [37]. The larger experimental sputtering rate, $5 \times 10^{-4}$ sputtered atoms per incident neutron [38], combined with a neutron flux of $3 \times 10^{20}$ neutrons $/ \mathrm{cm}^{2}$ year would give a forward sputtering rate of $5 \times 10^{16}$ atoms $/ \mathrm{cm}^{2}$.year. For backward sputtering, the expected rate is 0.06 of this value, but because neutrons impinge at grazing incidence, we will assume forward sputtering rates.

The gas shield required for protecting the mirror from soft $\mathrm{x}$ rays introduces an additional source of sputtering, gas atoms scattered into the mirror from neutron collisions. Argon atoms in the gas shield can receive up to 1.33 $\mathrm{MeV}$ from an elastically backscattered $14-\mathrm{MeV}$ neutron. The range of 1.33 - MeV Ar in Ar at 1 Torr is $2 \mathrm{~m}$ [39]. We therefore expect contributions to the sputtering from the entire gas shield. At $14 \mathrm{MeV}$, elastic scattering is strongly forward peaked. We expect the major contribution to be from nonelastic events with an average argon recoil energy of $\sim 0.33 \mathrm{MeV}$ and a cross section of 1 barn. We will assume an effective cross section of 1.5 barn for 0.33 - $\mathrm{MeV}$ recoils to account for elastic contributions and use 0.7 $\mathrm{m}$ as the appropriate range. This gives a bombardment rate of $3 \times 10^{-6}$ argon atoms per incident neutron.

A mirror $30 \mathrm{~m}$ from the reactor would therefore be exposed to $10^{15} \mathrm{Ar} / \mathrm{cm}^{2}$.year with energies from a few eV to $0.9 \mathrm{MeV}$. For $\mathrm{Al}$ and $\mathrm{Mg}$, the expected sputtering yields [40] are $\sim 3-4$ for Ar bombardmenı, so we expect $3 \times 10^{15}$ atoms $/ \mathrm{cm}^{2}$ year to be sputtered from the mirror by the argon atoms, which is only $6 \%$ of the maximum neutron sputtering rate. 
The atomic densities of magnesium and aluminum are approximately 4 and $6 \times 10^{22} \mathrm{~cm}^{-3}$ so the maximum erosion rates will be 36 and $24 \mathrm{~nm} /$ year, respectively. Although the annual erosion $(\lambda / 7$ or $\lambda / 10)$ is negligible from a riacroscopic viewpoint, only 1 to $2 \mathrm{~nm}$ is required to convert from specular to diffuse reflection from the electrons at the mirror surface. This change will increase the absorption of both $\mathrm{Al}$ and $\mathrm{Mg}$ by $0.35 \%$ after the first month of operation.

\section{Other Surface Damage:}

Surface cratering, which is produced by dense near-surface collision cascades in high- $Z$ metals, will not occur in magnesium or aluminum.

Surface pitting from voids is also expected to be negligible. Since the surface acts as a sink for defects, the region within $\sim 30$ to $50 \mathrm{~nm}$ of the surface is essentially free of voids.

\subsection{Neutron-Induced Swelling}

Neutron-induced swelling is expected to seriously affect the optical performance of the reflector. The tolerable swelling can be obtained from estimates of the nonuniform gradients in the mirror surface, $\delta l / l$, caused by swelling of the mirror material.

Optical distortion is a result of nonuniform gradients in $\delta l / l$ because a uniform gradient causes no distortion (although it will require pointing adjustments). For small fractional volume changes $(\delta V / V)$, the fractional linear dimension change is approximately given by:

$$
\delta l / l \simeq(1 / 3)(\delta V / V)
$$


The allowed deviation from a perfect plane for the mirror is $\lambda / g$, where $g$ is a scaling factor for optical quality. The figure, $\lambda / g$, is given by:

$$
\frac{\lambda}{g}=\mathrm{f} \delta l=\mathrm{f}(1 / 3)(\delta V / V) l
$$

where $\mathrm{f}$ is the fractional variation, $\delta l / l$, of the mirror surface relative to a plane tangent to the mirror at its center over distances much greater than $\lambda$ corresponding to figuring errors. Since the envisioned optical systems will be operated well away from the diffraction limit, with a focal spot diameter twenty times the diffraction limited value in a typical case, variations of the mirror surface from flatness up to $\lambda / 4$ are easily tolerable.

The variation $\mathrm{f}$ in $\delta l / l$ was estimated as follows. Since neutron fluence is proportional to $1 / R^{2}$, where $R$ is the distance from the pellet, and the void swelling is proportional to the neutron fluence, the swelling at any position relative to the center of the mirror is $\left(R / R_{\mathrm{c}}\right)^{2}$, where $R_{\mathrm{c}}$ is the distance from the pellet to the mirror center. For the mirror geometry given in section 6.1 , a slab $6.9 \times 1.8 \mathrm{~m}$ tilted $5^{\circ}$ from the beam, differential swelling will cause a slight tilt, concave curvature along the long axis and convex curvature transverse to the beam. From the center to the ends, $f$ is $+4 \times 10^{-4}$ and the transverse $\mathrm{f}$ is $-7 \times 10^{-4}$ for a total $\mathrm{f}$ of $11 \times 10^{-4}$, which is relatively small.

The allowed volumetric swelling can now be calculated from the allowable optical figure of merit, $\lambda / 4$. For light with $\lambda=250 \mathrm{~nm}$ and $l$ (optical surface and backing) $=12 \mathrm{~mm}$, the optically tolerable swelling is

$$
(\delta V / V)=3\left(\frac{\lambda}{4}\right)\left(\frac{1}{l f}\right)=1.5 \%
$$

This distortion corresponds to a shift of the focal point by $270 \mu \mathrm{m}$ due to tilting, an increase in spot dimensions of $170 \mu \mathrm{m}$ in the longitudinal direction and $20 \mu \mathrm{m}$ in the transverse direction. This is a conservative limit; the allowed swelling would be larger for longer $\lambda$ or smaller $g$. 
Aluminum and magnesium both exhibit significant void swelling at room temperature. In reactor irradiations, aluminum swells $\sim 1 \%$ for every $10^{21}$ $\mathrm{n} / \mathrm{cm}^{2}$ near room temperature, and magnesium swells three times as much [41]. Scaling these results on a damage energy basis, we expect to reach $1 \%$ swelling at $14-\mathrm{MeV}$ fluences of $\sim 2.5 \times 10^{20} \mathrm{n} / \mathrm{cm}^{2}$ in aluminum and $8 \times 10^{19}$ $\mathrm{n} / \mathrm{cm}^{2}$ in magnesium. We would thus expect a lifetime limit (due to $1.5 \%$ volumetric swelling) for the mirror of 1.2 full-power years for aluminum and 0.4 full-power years for magnesium. For alloyed aluminum, the onset of swelling in reactor irradiations is delayed to about $2 \times 10^{21} \mathrm{n} / \mathrm{cm}^{2}$, which would increase the lifetime to 2.4 full-power years [42]. At LN temperatures, void swelling does not occur. The point defect saturation that does occur would produce an insignificant 0.3 to $0.5 \%$ volumetric expansion.

\subsection{Other Radiation Damage and Heating}

Extremely effective shielding of the first mirror from atomic particles (p, d, and $\alpha$ ) is required to prevent large increases in the optical absorptance and damage by blistering, sputtering or heating. Fortunately, atomic particles are stopped in the target for indirect drive targets and possibly for direct drive targets as well. Shielding the mirror from $\mathrm{x}$ rays will be required to prevent melting if $\sim 0.3 \%$ or greater of the pulse energy is in cold $(\hbar \omega \simeq 1$ $\mathrm{keV}$ ) $\mathrm{x}$ rays. One Torr-m of argon is sufficient to shield the mirror from cold $x$ rays. Neutrons and energetic macroscopic fragments will not be stopped by 1 Torr-m of gas. A high-speed shutter is required to block macroscopic projectiles.

\subsection{Mirror Lifetime Radiation Damage Limit}

Successful operation of a magnesium or aluminum mirror located $30 \mathrm{~m}$ from a $1000-\mathrm{MJ}, 3-\mathrm{Hz}$ pellet is theoretically possible. The mirror must be shielded from $\alpha$ particles, other charged particles, short-range neutral particles, macroscopic pellet debris, and $\mathrm{x}$ rays. For high $\rho R$ targets, less than 1 Torr-m of argon provides sufficient shielding for everything except the slow- 
moring pellet debris. Neutron damage will increase the optical absorptance by a factor of at most 2 , thus lowering the laser-damage threshold by a factor of approximately 2. A clean aluminum alloy mirror operated below this threshold should have a fluence limit of $10^{21} \mathrm{n} / \mathrm{cm}^{2}$ or 2.4 full-power years in a $1000-\mathrm{MW}_{\mathrm{e}}$ plant. Overall swelling, melting, vaporization, surface erosion, creep, and dimensional and mechanical instability as well as increased absorptance from transmutation products and neutron-induced defects are expected to be tolerable.

\section{Cryogenic Operation of Metal Mirrors}

For most metals, cooling the mirror to $\mathrm{LN}$ temperatures offers several advantages. In addition to the elimination of void swelling, the absorptance of an undamaged cryogenic $(\sim 77 \mathrm{~K})$ mirror made of pure magnesium or aluminum is lower than that of a similar mirror operated at room temperature. The damage threshold of the mirror due to thermal stresses is also raised, since both the thermal conductivity and the fatigue stress of the metals increase at lower temperatures and the thermal expansion decreases. These advantages must be balanced against the added complexity and cost of cryogenic operation. An aluminum alloy mirror (made of $\mathrm{Al}$ 7475-T7351 or Al 6061-T6) might be operated at room temperature, while one of pure aluminum or magnesium would require cryogenic operation.

The retention of damage at $77 \mathrm{~K}$, which saturates in the first few weeks of operation, will just compensate for the temperature reduction in absorption, but the saturation damage will also lower the thermal conductivity. However, the improved fatigue stress and the lowered expansion rate still result in a net increase of the damage limit. A further improvement in the damage limit results from an increase of the fatigue stress due to radiation hardening. Radiation hardening increases the ultimate tensile stress of aluminum and its alloys by 90 to $100 \mathrm{MPa}$ [43]. This would lead us to expect an increase of 45 to $50 \mathrm{MPa}$ in the fatigue limit of aluminum. If the hardening mechanisms are similar in magnesium, we would expect an increase in the fatigue stress of $20 \mathrm{MPa}$. Because of the short duration of the ther- 
mal pulse, annealing of the radiation-induced resistivity and mechanical property changes at $77 \mathrm{~K}$ will not occur [44] unless the peak temperature exceeds $300 \mathrm{~K}$.

The calculation of these limits is discussed in the next section of this paper. Using mirrors that are initially at $\mathrm{LN}$ temperatures increases the damage threshold of pure metal mirrors by a factor of 2 to 3 . The damage thresholds for the representative illumination geometry (caliulated in section 5.3) for irradiated aluminum, magnesium, and aluminum alloys at $77 \mathrm{~K}$ are, respectively, $1.0,0.65$, and 1.65 to $2.25 \mathrm{~J} / \mathrm{cm}^{2}$ of beam energy. 


\section{Surface Temperature Rise and Laser Dam- age Limits for Irradiated Metal Mirrors}

\section{Surface Temperature Rise for the Mirror:}

The surface temperature rise for a heat load deposited on the surface of a metal mirror is given by:

$$
\Delta T=\frac{2 q_{0}}{k_{\mathrm{T}}}\left(\frac{\alpha t}{\pi}\right)^{1 / 2}
$$

for

$$
\alpha=\frac{k_{T}}{\rho c}
$$

where:

$q_{0}=$ surface power density given by $q_{0}=(1-R) q_{\text {beam }}$, where

$q_{\text {beam }}=$ the instantaneous power density of the optical beam;

$R=$ reflectance of the mirror for a given angle of incidence of the light;

$t=$ time duration of the pulse;

$\rho=$ density of the metal;

$\alpha=$ thermal diffusivity;

$k_{\mathrm{T}}=$ thermal cunductivity;

$c=$ heat capacity of the metal.

In section 6.3, the maximum allowable temperature rise is found from thermal stress limits and used to find a maximum allowable power density, $q_{0}=(1-R) q_{\text {beam }}$, and a corresponding minimum possible mirror area. Table 4 shows the results of these calculations for grazing incidence light ( $85 \%$ angle of incidence). The need to control the surface temperature rise is the primary reason for using grazing incidence reflectivity. 
Table 4 Damage limits for aluminum and magnesium mirrors

\begin{tabular}{|c|c|c|c|c|c|c|c|c|}
\hline Metal & $\mathrm{Mg}$ & Mg & All 100-O & Al1 100-O & Al6061-T6 & Al6061-T6 & A17475-T7351 & A17475-T7351 \\
\hline$T_{0}$ & 293 & 77 & 293 & 77 & 293 & 77 & 293 & 77 \\
\hline$T_{u}$ & 400 & 293 & 400 & 293 & 400 & 293 & 400 & 293 \\
\hline Fatigue stress $\left(10^{8} \mathrm{~Pa}\right)$ & 0.340 & 0.800 & 0.430 & 1.40 & 1.80 & 3.10 & 2.90 & 3.95 \\
\hline Poisson's ratio & 0.29 & 0.28 & 0.33 & 0.32 & 0.33 & 0.32 & 0.33 & 0.32 \\
\hline Energy $\left(10^{10} \mathrm{~N} / \mathrm{m}^{2}\right)$ & 4.45 & 4.89 & 7.00 & 7.75 & 6.97 & 7.71 & 7.30 & 8.03 \\
\hline$R$ & 0.9899 & 0.99 & 0.9898 & 0.9899 & 0.9895 & 0.9896 & 0.9894 & 0.9895 \\
\hline$\delta 1 / I_{\max }(\%)$ & 0.0542 & 0.1178 & 0.0412 & 0.1228 & 0.1730 & 0.2734 & 0.2662 & 0.3345 \\
\hline$a_{1}$ & 340.1 & 347.7 & 377.4 & 318.5 & 365.7 & 355.2 & 338.2 & 399.1 \\
\hline$a_{2}$ & 21.4 & 98.2 & 18.2 & 145.6 & 28 & 116.2 & 39.6 & 89.9 \\
\hline$\delta T_{\max }$ & 23.43 & 74.66 & 19.22 & 90.15 & 74.92 & 157.88 & 110.45 & 185.49 \\
\hline$l$ & 22.6 & 22.6 & 22.1 & 22.1 & 22.8 & 22.8 & 22.8 & 22.8 \\
\hline p20 & 44.5 & 55.5 & 29.2 & 39.2 & 40 & 50 & 41.1 & 51.1 \\
\hline$d \rho / d T$ & 0.157 & 0.157 & 0.115 & 0.115 & 0.115 & 0.115 & 0.115 & 0.115 \\
\hline$\rho c$ & 46 & 46 & 33.9 & 33.9 & 33.9 & 33.9 & 33.9 & 33.9 \\
\hline ko & 148.80 & 80.61 & 223.32 & 120.22 & 167.87 & 70.35 & 163.35 & 67.38 \\
\hline$k m$ & 148.43 & 102.90 & 221.12 & 150.64 & 173.28 & 124.22 & 171.63 & 126.30 \\
\hline$k u$ & 147.47 & 119.31 & 214.04 & 166.05 & 175.05 & 134.16 & 171.43 & 131.26 \\
\hline$p c_{0}\left(10^{6}\right)$ & 1.78 & 0.859 & 2.45 & 0.928 & 2.42 & 0.916 & 2.42 & 1.40 \\
\hline$\rho c_{u}\left(10^{6}\right)$ & 1.88 & 1.78 & 2.51 & 2.45 & 2.48 & 2.42 & 2.48 & 2.42 \\
\hline $\operatorname{del} \theta\left(10^{2}\right)$ & 0.234 & 0.865 & 0.191 & 1.04 & 0.762 & 2.29 & 1.13 & 2.83 \\
\hline$\langle<\rangle\left(10^{-5}\right)$ & 8.30 & 8.92 & 9.06 & 1.17 & 6.98 & 6.90 & 6.83 & 5.08 \\
\hline$q_{\max }\left(10^{10} \mathrm{~W} / \mathrm{m}^{2}\right)$ & 0.339 & 0.654 & 0.398 & $1 . n_{-}^{\prime}$ & 1.36 & 1.72 & 1.99 & 2.37 \\
\hline$E_{\text {bearnoux }}\left(J / \mathrm{cm}^{2}\right)$ & 0.34 & 0.65 & 0.39 & 1.01 & 1.29 & 1.65 & 1.88 & 2.26 \\
\hline$A_{\min }\left(\mathrm{m}^{2}\right)$ & 46.59 & 23.89 & 40.08 & 15.44 & 12.09 & 9.44 & 8.33 & 6.93 \\
\hline
\end{tabular}

\subsection{Comparison of Optical Penetration Depth with Thermal Profile Depth and Atomic Separation Distances in the Metal}

For the above calculations for surface temperature rise to be valid, the optical penetration depth must be small relative to the thermal profile depth and large relative to the atomic separation distances in the metal. 
The optical penetration distance is given by:

$$
d=\frac{\lambda_{0}}{4 \pi k}
$$

where $k$ is the extinction coefficient of the metal. For 353-nm light, the penetration distances are $10 \mathrm{~nm}$ for magnesium and $7.2 \mathrm{~nm}$ for aluminum. Because this distance is a $1 / e$ folding distance, the penetration depth will be equal to $d \sin 5^{\circ}$ or $0.087 d$.

The depth of the thermal profile after $t$ seconds is given by:

$$
d_{t}=\left(\frac{k_{\mathrm{T}} t}{\rho c}\right)^{1 / 2}
$$

where $k_{\mathrm{T}}, \rho$, and $c$ are the material constants previously defined.

In $10 \mathrm{~ns}$, the thermal profile has moved to a depth of $0.93 \mu \mathrm{m}$ for aluminum. The assumption that the heat can be treated as being generated entirely on the surface of the metal will remain valid as long as this depth is significantly longer than the optical penetration depth. Since the pulse length for which they are equal is on the order of $10^{-5} \mathrm{~ns}$, this should be a reasonable assumption for any anticipated pulse lengths.

\subsection{Effect of Pulse Shaping Gin Surface Temperature Rise}

The previous calculations assumed a flat 10-ns pulse. If a shaped pulse is used, the temperature rise will be different. For a two-plateau, 5-MJ pulse (Fig. 6) with a $30-n s$ plateau containing $30 \%$ of the pulse energy followed by a 5 -ns plateau containing $70 \%$ of the pulse energy, the total surface temperature rise can be assumed to be approximately given by the sum of the temperature rises given by each plateau. For a cryogenic mirror with constant material properties equal to their values at $77 \mathrm{~K}$, the temperature rise from this two-plateau pulse is only $17 \%$ higher than the 
temperature rise of a 10-ns flat pulse with the same total energy. The surface temperature rise due to the 30 -ns low-intensity pulse is sufficiently small that the mirror would still have the benefit of cryogenic properties when the high-intensity pulse began. The mirror limits for a pulse with extreme pulse shaping will be only slightly more restricting than those for a flat pulse.

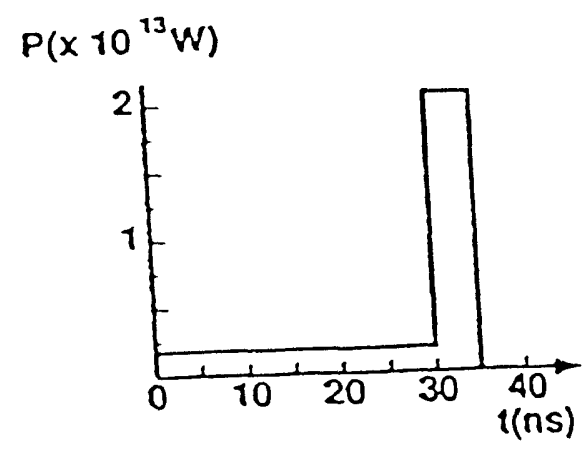

Figure $6 \mathrm{~A}$ two plateau shaped pulse delivering $5 \mathrm{MJ}$ to the target.

\subsection{Laser Damage Limits for Irradiated Metal Mir- rors}

The reflectivities for the damaged mirrors are used with thermal stress limits for a cyclic load to give the maximum allowable beam energy density and corresponding minimum mirror size 10 r each of 32 beams delivering a total of $5 \mathrm{MJ}$ of beam energy in $10 \mathrm{~ns}$. The resulting damage thresholds and mirror sizes are shown in Table 4.

If the mirror surface can be kept clean and unpitted, the most limiting constraint on the allowed mirror size will be that due to the thermal stress caused by the surface temperature rise. For any material free to expand in one direction and completely constrained in the other two, the thermal stress is given by: 


$$
\sigma_{\text {thermal }}=\left(\frac{E}{1-\nu}\right) \frac{\Delta l-(\Delta l)_{\text {ave }}}{l}
$$

where $E$ is Young's modulus, $\nu$ is Poisson's ratio, $l$ is the length along the free dimension, $\Delta l$ is the expansion length, and $(\Delta l)_{\text {ave }} / l$ is the volumeaveraged elongation of the material. For a 10 -ns pulse, $\Delta l$ is negligible. For a flat pulse with step heat load, $q$, applied for a duration $t_{\text {shot }}$, the temperature rise, $\Delta T$, as a function of time, $t$, and position, $x$, will be:

$$
\begin{gathered}
\Delta T=2 q \sqrt{\frac{t}{k_{\mathrm{T}} \rho c}}\left[\frac{\exp \left(-F o_{x}^{*^{2}}\right)}{\sqrt{\pi}}-F o_{x}^{*} \operatorname{erfc}\left(F o_{x}^{*}\right)\right], \text { for } t<t_{\text {shot }} \\
\Delta T=q \sqrt{\frac{t}{k_{\mathrm{T}} \rho c}}\left\{2 \operatorname{ierfc}\left(F o_{x}^{*}\right)-2 \sqrt{\frac{t-t_{\text {shot }}}{t}} \operatorname{ierfc}\left[\frac{x}{2 \sqrt{\frac{k\left(t-t_{\text {shot }}\right)}{\rho c}}}\right]\right\}, \text { for } t>t_{\text {shot }}
\end{gathered}
$$

where:

$F o_{x}^{*}=\frac{x \sqrt{\rho c}}{2 \sqrt{k_{\mathrm{T}} t}}$ for $\rho, k_{\mathrm{T}}$, and $c$ as defined before;

erfc $=$ complementary error function;

ierfc $=$ integral of the complementary error function. 
If we require that the thermal stress be less than the fatigue stress, $\sigma_{f}$, of the material, we must limit the surface expansion to:

$$
\left(\frac{\Delta l}{l}\right)_{\max }=\frac{\sigma_{\mathrm{f}}(1-\nu)}{E}
$$

Since expansion is a function of temperature rise, the maximum allowable temperature rise can be determined from the maximum permissible expansion. For a fiven material, this maximum temperature rise, $\Delta T_{\max }$ will correspond to a maximum allowable heat load given by:

$$
q_{\max }=(\sqrt{\pi} / 2) k \sqrt{\frac{1}{\alpha t_{\text {shot }}}} \Delta T_{\max }
$$

where $\alpha$ is the linear coefficient of thermal expansion. Multiplying this maximum heat load by $10^{-8} \mathrm{~s}$ gives the maximum allowable energy density, $E_{\max }\left(\right.$ in $\mathrm{J} / \mathrm{m}^{2}$ ), for the mirror. The minimum mirror size for a mirror is then given by:

$$
A_{\min }=\left[\frac{(1-R) \times 5 \mathrm{MJ}}{32}\right]\left(\frac{1}{E_{\max }}\right) .
$$

The above calculations must be modified to account for the temperature dependence of $k, \rho$, and $c$. In general, closed solutions for the temperature dependent equations are not available, but a solution does exist if we assume constant $\alpha$ and variable $k$ [45]. In this case, the above solutions may be used by substituting $\Delta \theta$ for $\Delta T$, where $\Delta \theta=\left(1 / k_{0}\right) \times \int_{0}^{\Delta T} k d(\Delta T)$ for $k_{0}=k(\Delta T=0)$. The assumption of constant $\alpha$ is good to better than $20 \%$ for all of the materials and temperature ranges considered here. The temperature dependence of $\mathrm{k}$ was dete $\mathrm{min}^{-1}$ from the electrical resistivity changes and the Wiedemann-Franz la' $k, k=L T / \rho$, where $L$ is the Lorenz number. The thermal, electrical, and elastic properties for the metals were taken from the Metals Handbook [46]. Fatigue limits were taken from the literature $[47,48]$. The temperature dependence of the resistivity, thermal expansion, and specific heat of the metals was obtained from Thermophysical Properties of Matter [49]. 
Table 4 lists the results of these calculations for aluminum, magnesium, and alloyed aluminum at $\mathrm{LN}$ and room temperatures. These values assume $5-\mathrm{eV}$, or $250-\mathrm{nm}$, photons. For $2.5-\mathrm{eV}$, or $500-\mathrm{nm}$, photons, the limiting mirror sizes are $10 \%$ larger for aluminum and its alloys, but the limiting sizes of magnesium mirrors are $20 \%$ smaller. Although cryogenic operation reduces the required mirror size for pure aluminum and magnesium by a factor of 2 to 3 , for the alloys the reduction in mirror size is only $20 \%$ for the best candidate (A17475-T7351). Because of the increased cost and complexity of LN cooling, a room-temperature aluminum alloy mirror is the most desirable choice.

While the largest gains in fatigue stresses are for precipitation-hardened aluminum alloys, such as we have described here, they may not prove to be usable for two reasons:

1. If the second phase (typically a few percent) has a large absorption coefficient, the gains may be nullified.

2. At room temperature, the mass transport occurring during defect annihilation processes may cause over-ageing of the alloys and a subsequent lowering of the fatigue stress.

If either of these concerns is prohibitive, we could still use slightly larger mirrors made of a solid-solution wrought alloy. 


\subsection{Heat Removal Requirements for Metal Mirrors}

For any illumination geometry similar to that shown in Fig. 2, the energy absorbed from the laser beams at the mirror surface will be much larger than that from any other source of heat. For $5 \mathrm{MJ}$ of total energy to be delivered by 32 beams, each beam must have $156 \mathrm{~kJ}$ of energy. This means that a mirror with a reflectance of $99 \%$ will absorb $(1-R) E_{\text {beam }}=1.56$ $\mathrm{kJ} / \mathrm{shot}$. For a repetition rate of $3 \mathrm{Hertz}$ and a conservative limit on the mirror size of $5 \mathrm{~m}^{2}$, this gives a required heat removal rate of $0.15 \mathrm{~W} / \mathrm{cm}^{2}$, which corresponds to a required average temperature gradient of $0.07 \mathrm{~K} / \mathrm{cm}$ for $\mathrm{Al}$. For a cooling channel located $2 \mathrm{~mm}$ below the surface of the mirror, this requires an average temperature difference of $0.013 \mathrm{~K}$ between the surface and the coolant. The thermal expansion that accompanies this temperature difference in aluminum is given by $\Delta l=\alpha \Delta T \times 2 \mathrm{~mm}$, or $0.6 \mathrm{~nm}$. The mirror distortion will propagate at the sound speed, so the pulse will be finished before the mirror has time to distort. Even if the maximum distortion affected the beam, the warping moment associated with the thermal stresses acts over a thin enough depth (the depth of the thermal profile at the end of $10 \mathrm{~ns}$ is approximately $1 \mu \mathrm{m}$ ) that the coating on a 1 - to $2-\mathrm{cm}$-thick mirror would not undergo the $1-\mu \mathrm{m} / \mathrm{m}$ deflection required to defocus the beam.

\section{Beam Focusing and Required Mirror Geometry}

\subsection{Mirror Dimensions}

For a 1- $\times 3$-m elliptical final dielectric mirror (3 times the area required for a $20-\mathrm{J} / \mathrm{cm}^{2}$ damage limit) located $50 \mathrm{~m}$ from the target and a flat mirror located $30 \mathrm{~m}$ from the target at an angle of $5^{\circ}$ to the beamline, the flat mirror would have to be $6.9 \times 1.8 \mathrm{~m}$ to reflect the entire beam. This would give a mirror area of $10 \mathrm{~m}^{2}$, which is large enough to operate a room-temperature aluminum alloy mirror below its damage limit. 
For larger mirror sizes, it may be more practical to use segmented mirrors (ten $1-\mathrm{m}^{2}$ mirrors instead of one $10-\mathrm{m}^{2}$ mirror). Grazing incidence light and rounded mirror edges may avoid edge effect problems, which would be significant in normal incidence mirrors.

\subsection{Beam Pointing}

Because of the small cone angles and long focal lengths of the driver optics, the focal spot size is relatively insensitive to the distance between the final nirror and the target. To compensate for variations in target positioning between shots, refocusing is not necessary, but two-dimensional beam pointing is essential. This pointing can be done by two-axis rotation of either the final dielectric mirror or the grazing incidence metal mirror.

If all final pointing adjustments are made at the metal mirror, then the mirror must be able to rotate about, both facial axes. If the target position at the time of the shot varies by $1 \mathrm{~mm}$ in any direction, the required rotation around the shorter facial axis is approximately $\delta \theta_{1}=0.001 \mathrm{~m} / 30 \mathrm{~m}=3.33$ $\times 10^{-5}$ radians or about $2 \times 10^{-3}$ degrees, and the required rotation about the longest mirror axis is approximately $0.001 \mathrm{~m} /\left(30 \mathrm{~m} \sin 5^{\circ}=0.219\right.$ radians or 1.25 degrees. Because these adjustments are so small, they can be made quickly with a small amount of power. A 12-mm-thick, 6.9- $\times 1.8$-m mirror can make either adjustment in $0.1 \mathrm{~s}$ with a few watts of applied power.

The most critical issue in pointing with the metal mirror is the need for high-accuracy mirror positioning and accurate position variations over very small distances. A deflection of one end of the mirror by $1 \mathrm{~mm}$ will shift the focal spot by $1 \mathrm{~cm}$ in the target plane. Any mechanism for mirror steering must be carefully designed so it does not distort the optical surface as it moves the mirror. 


\section{Critical Issues and Areas for Further Work}

From considerations of neutron damage, reflectivity, heat removal, and surface temperature rise, grazing incidence metal mirrors appear to be very attractive. The most crucial future work needed to verify the integrity of these mirrors includes:

- Experimental verification of laser damage thresholds

A small-scale experiment could be done with small undamaged mirrors to verify the calculated normal and grazing incidence reflectivities and to measure the effect of oxide coatings on reflectivity for ec ch of the candidate metals. The effect of an oxidation layer on the normal incidence $353 \mathrm{~nm}$ reflectivity of $\mathrm{Al}$ or $\mathrm{Mg}$ is small (a fraction of a percent) or nonexistent, but the effect may be larger for grazing incidence. A Los Alamos study of Al grazing incidence mirrors[50] showed significant degradation of the reflectivity for light with wavelengths below $100 \mathrm{~nm}$.

- Experiments with irradiated mirrors:

The laser damage experiments could be repeated with srnall irradiated samples to verify the effects of neutron irradiation on the laser damage threshold. Although it may be difficult to get a sufficient source of $14 \mathrm{MeV}$ neutrons, fission reactor irradiations could be used and the results scaled with total damage energy.

- Protection of the final mirror from debris and $x$-rays

Even if the heat loads on a perfectly smooth and clean mirror are low enough to avoid surface vaporization, a particle or surface defect on the mirror surface would be exposed to the full normal incidence beam energy and could cause explosive "pitting" of the mirror surface. Accumulation of material (target debris, Flibe, or other coolant/breeder materials) from the reaction chamber on the mirror surface must be prevented. Gas jets used for protection from x-rays will not provide sufficient protection from high-velocity debris. There is a need for 
a high-speed mechanical shutter[51] to protect the mirror. Both the gas and shutter systems require significant design work before their effectiveness can be judged.

- Development of techniques for cleaning the final mirrors

If a combination of high-speed shutters and gas jets can protect the mirror surface from damage but cannot keep it clean, it may be possible to remove accumulated material from the mirror surface between shots. A possible technique would be to vaporize the contaminants with a lower energy beam between shots.

- Manufacturing studies for large high-quality mirrors

Large-scale high-quality mirrors can be manufactured, but the cost of producing $10-\mathrm{m}^{2}$ mirrors with surfaces finished to tens of nanometers may be prohibitive.

Other concerns that are independent of future mirror work are:

- The effect of large mirror cooling requirements on system efficiency

The energy required to cool a room-temperature mirror is not large, but cooling a cryogenic mirror could require enough energy to significantly affect the plant power balance. Removing $1 \%$ of the total beam power from the final mirrors at $77 \mathrm{~K}$ could require tens to hundreds of megawatts of recirculating power. Since low laser-driver efficiencies are a critical problem, the cost of additional recirculating power may be unacceptable.

- Nonuniform beam intensity

Nonuniform beam intensity could force the use of larger mirrors. Multiplexed beams from $\mathrm{KrF}$ lasers will not only have nonuniformities, they will also be coming from different angles of incidence. The best achievable peak-to-average power ratios at the final amplifier of Nova are typically 1.4-1.6. Streak camera profiles of a Nova beam immediately after polarization and frequency conversion show a lower peak-to-average power ratio, with spike widths on the order of a millimeter. Transverse heat flow and stress release during a 10-ns shot in the mirror can only average out nonuniformities over distances of 
a micron or less, so peaks wider than a micron will lead to local failure unless a larger mirror is used. Beam nonuniformity grows as the beam converges, so nonuniformities at the mirror will be worse than those after the final focusing or conversion element.

- Brightness requirements for $\mathrm{KrF}$ lasers may require a minimum solid angle illumination which would correspond to mirrors much larger than those which are damage limited. A required total illumination solid angle of $0.04 \pi$ steradians would require a grazing incidence mirror size of $40 \mathrm{~m}^{2}$ for each mirror in a 32 -mirror system.

\section{Conclusions}

Preliminary calculations indicate that grazing incidence metal mirrors appear to offer a solution to the critical problem of neutron damage to beam line components. A final mirror made of $\mathrm{Al}$ alloy could be used for 2.4 years at room temperature before replacement and for much longer times at $77 \mathrm{~K}$. Larger mirrors made of pure $\mathrm{Al}$ or $\mathrm{Mg}$ could also be used at $77 \mathrm{~K}$. Although there are important concerns which have yet to be investigated, metal mirrors may provide a solution to the crucial problem of how to interface a laser driver with an ICF reactor. Solving this problem is essential if lasers are to be credible driver candidates for ICF power plants. 


\section{References}

[1] B. E. NEUNAM, "Multifacet Metal Mirror Design for Soft X-Ray and Extreme-Ultraviolet Free-Electron Laser Resonators," in Laser Induced Damage in Optical Materials: 1985, H. E. BENNETT, A. H. GUENTHER, D. MILAM, and B. E. NEWNAM, Eds. (National Bureau of Standards, Washington, DC), Special Publication 746, (1988), pp. 261-269.

[2] R. L. BIERI and M. W. GUINAN, "An Analysis of Grazing Incidence Metal Mirrors in a Laser ICF Reactor Driver,"UCRL-JC106094,Lawrence Livermore National Laboratory preprint (1990).

[3] M. TOBIN and R. BIERI, "Analysis of the Grazing Incidence Metal Mirror (GIMM) Concept for HYLIFE-II," internal LLNL HYLIFE-II memo (1990).

[4] M. J. WEBER, Handbook of Laser Science and Technology, Vol. III, Optical Materials: Part 1, 336 and 366, CRC Press, Inc. (1986).

[5] F.W. CLINARD, JR., and L. W. HOBBS, "Radiation Effects in NonMetals," in Physics of Radiation Effects in Crystals, 225-280, Eds. R.A. Johnson and A.N. Orlov, North Holland, Amsterdam (1986).

[6] H. WIDERSICH, "Phase Stability and Solute Segregation during Irradiation," ibid, 387-471.

[7] R. KINGSLAKE, Ed., Applied Optics and Optical Engineering, Vol. III, Optical Components, 317, Academic Press, N.Y. and London (1965).

[8] G. HASS, "Optical Properties of Metals," in AIP Handbook, 3rd ed., B. H. Billings, Ed. (McGraw-Hill, New York, 1972), ch. 6 pp. 118-169.

[9] C. L. FOILES, "Optical Properties of Pure Metals and Binary Alloys," in Landolt-Bornstein, New Series III, vol. 15b, K.-H. Hellwege and O. Madelung, Eds. (Springer-Verlag, New York, 1985), ch. 4.

[10] G. HASS and J. E. WAYLONIS, J. Opt. Soc. Am. 44, 357 (1954). 
[11] C. J. POWELL, J. Opt. Soc. Am. 60, 78 (1970).

[12] H.-J. HAGEMANN, W. GUDAT, and C. KUNZ, DESYSR-74/7 Hamburg, (1974).

[13] P. B. JOHNSON and R. W. CHRISTY, Phys. Rev. B6, 4370 (1972).

[14] C. J. FLATEN and E. A. STERN, Phys. Rev. B11, 638 (1975).

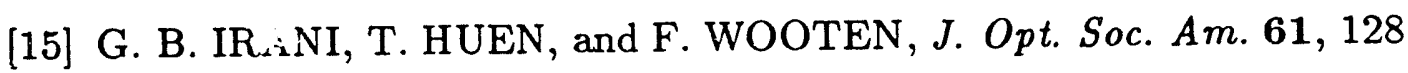
(1971).

[16] R. H. HUEBNER, E. T. ARAKAWA, R. A. MACRAE, and R. N. HAMM, J. Jpt. Soc. Am. 54, 1434 (1964).

[17] H. G. LILJENVALL and A. G. MATHEWSON, J. Phys. C: Metal Phys. 3, S341 (1970).

[18] P. DOBBERSTEIN, A. HAMPE, and G. SAUERBREY, Phys. Lett. 27 A, 256 (1968).

[19] T. HUEN, B. B. IRANI, and F. WOOTEN, Appl. Opt. 10, 552 (1971).

[20] O. HUNDERI and H. P. MYERS, J. Phys. F: Metal Phys. 3, 683 (1973).

[21, H. E. BENNETT and J. M. BENNETT, in Optical Properties and Electronic Structure of Metals and Alloys, F. Abeles, Ed. (J. Wiley, New York, 1966).

[22] F. WOOTEN, Optical Properties of Solids (Academic Press, New York, 1972), ch. 4.

[23] T. F. GESELL, E. T. AIAKAWA, M. W. WILlIAMS, and R. N. HAMM, Phys. Rev. B7, 5141 (1973).

[24] G. T. MEADEN, Electrical Resistance of Metals (Plenum Press, New York, 1965), ch. 5.

[25] A. V. SOKOLOV, Optical Properties of Metals (American Elsevier, New York, 1967), ch. 8. 
[26] T. HOLSTEIN, Phys. Rev. 96, 535 (1954).

[27] O. K. HARLING aid M.T. THOMAS in Proceedings of the 2nd Topical Meeting on Technology of Controlled Nuclear Fusion (USERDA Report CONF-760935-P1) Vol. 1, 149-167, (1976).

[28] H. H. ANDERSEN and H. L. BAY, in Sputtering by Particle Bombardment I, Topics in Applied Physics, 47, ed. R. Behrisch, 167-168, Springer-Verlag, Berlin (1981).

[29] H. E. BENNETT, "Optical Figure Requirements for Laser Mirrors Used at Oblique Incidence," in Laser Induced Damage in Optical Materials: 1982, H. E. Bennett, A. H. Guenther, D. Milam, and B. E. Newnam, Eds. (National Bureau of Standards, Washington, DC), Special Publication 669 (1984), pp. 228-233.

[30] L. R. GREENWOOD and R. K. SMITHERS, Specter: Neutron Damage Calculatione for Materials Irradiations (Argonne National Laboratory, Argonne, IL), Report ANL/FPP/TM-197 (1985).

[31] M. W. GUINAN, MRS Bull. XIV(7), 20ff (1989).

[32] P. JUNG, Phys. Rev. B23, 664 (1981).

[33] C. E. KLABUNDE and R. R. COLTMAN, JR., J. Nucl. Mater. 108, 109, 183 (1982).

[34] M. NAKAGAWA, J. Nucl. Mater. 108, 109, 194 (1982).

[35] R. L. BENBOW and D. W. LYNCH, Phys. Rev. B12, 5615 (1975).

[36] P. SIGMUND, Phys. Rev. 184, 383 (1969).

[37] M. T. ROBINSON, J. Nucl. Mater. 53, 201 (1974).

[38] O. K. HARLING and M. T. THOMAS in Proc. 2nd Topical Meeting on Technology of Controlled Nuclear Fusion, Vol. 1 (USERDA Repport CONF-760935-P1, 1976), pp. 149-167.

[39] L. C. NORTHCLIFFE and R. F. SCHILLING, Nuclear Data Tables A7, 233 (1970). 
[40] H. H. ANDERSEN and H. L. BAY, "Sputtering by Particle Bombardment I," in Topics in Applied Physics, Vol. 47, R. Behrisch, Ed. (Springer-Verlag, Berlin, 1981), ch. 4, pp. 167-168.

[41] Y. ADDA, "Radiation Induced Voids in Metals," in Proc., J. W. Corbett and L. C. Ianniello, Eds., NTIS Conf.-710601 (1972), p. 31.

[42] A. RISBET, Etude de la Formation des Cavites dans l'Aluminum Pur et Faiblement Allie Irradie aux Neutrons Rapides, CEN de Saclay Rapport CEA-R-4633 (1975).

[43] D. S. BILLINGTON and J. S. CRAWFORD, JR., Radiation Damage in Solids (Princeton University Press, Princeton, NJ, 1961), ch. 5, pp. 134-146.

[44] A. W. MCREYNOLDS, W. AUGUSTYNIAK, M. MCKEOWN, and D. B. ROSENBLATT, Phys. Rev. 98, 418 (1955).

[45] H. S. CARSLAW and J. C. JAEGER, Conduction of Heat in Solids, 2nd ed. (Clarendon Press, Oxford, 1986), pp. 10-13.

[46] Metals Handbook, 9th ed., vol. 2 (ASM, Metals Park, OH, 1979).

[47] R. D. MCCAMMON and H. M. RONSENBERG, "The Fatigue and Ultimate Tensile Strengths of Metals between 4.2 and $293^{\circ} \mathrm{K}$," Proc. Roy. Soc. London A242, 203 (1957).

[48] Aerospace Structural Metals Handbook, 1990 ed., vol. 3, (Army Materials and Mechanics Research Center, Watertown, MA, 1975), AFMLTR-68-115.

[49] Thermophysical Properties of Matter, vol. 4, 12, Y.S. Touloukian, Ed. (Plenum, New York, 1970, 1975).

[50] B.E. NEWNAM, "Multifacet Metal Mirror Design for Soft X-Ray and Extreme-Ultraviolet Free-Electron Laser Resonators," in Laser Induced Damage in Optical Materials: 1985, H.E. Bennett, A.H. Guenther, D. Milam, and B.E. Newnam, Eds., (Natl. Bur. Stand., Washington D.C.), NBS Spec. Publ. 746, 261-269, 1988. 
[51] D. G. NILSON and J. G. WOODWORTH, "Final Optic Protection Designs for ICF Containment Chambers," in Proceedings of the Eigth Topical Meeting on The Technology of Fusion Energy, Salt Lake City, Utah (1988). 

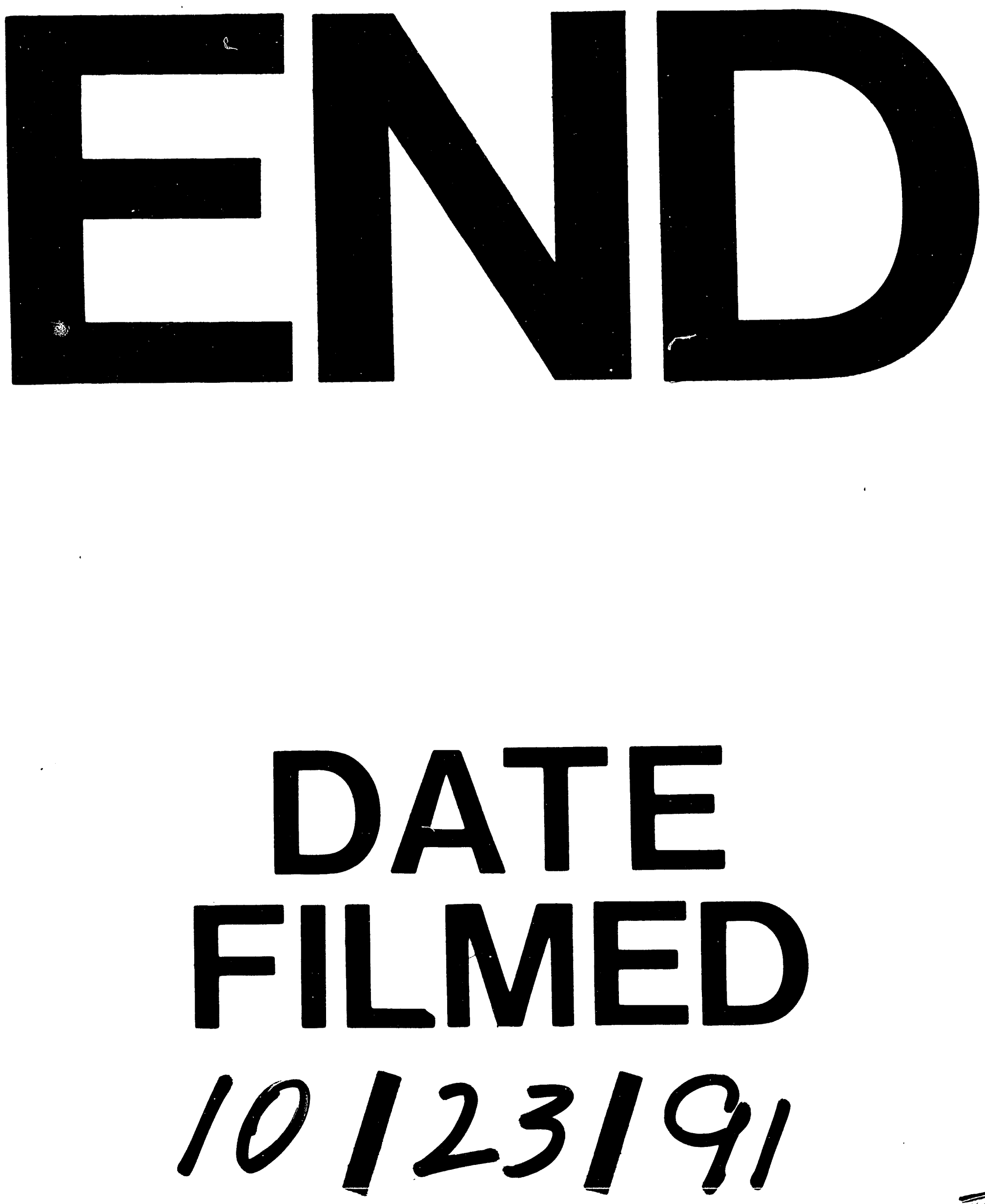


\section{,}

